\title{
OPEN Distribution and genome structures of temperate phages in acetic acid bacteria
}

\author{
Koki Omata ${ }^{1}$, Naruhiro Hibi ${ }^{2}$, Shigeru Nakano ${ }^{2}$, Shuji Komoto ${ }^{2}$, Kazuki Sato ${ }^{1}$, \\ Kei Nunokawaa ${ }^{1}$, Shoichi Amano ${ }^{1}$, Kenji Ueda ${ }^{1}$ \& Hideaki Takano ${ }^{1 凶}$
}

Acetic acid bacteria (AAB) are industrial microorganisms used for vinegar fermentation. Herein, we investigated the distribution and genome structures of mitomycin $\mathrm{C}$-inducible temperate phages in AAB. Transmission electron microscopy analysis revealed phage-like particles in 15 out of a total 177 acetic acid bacterial strains, all of which showed morphology similar to myoviridae-type phage. The complete genome sequences of the six phages derived from three strains each of Acetobacter and Komagataeibacter strains were determined, harboring a genome size ranging from 34,100 to 53,798 bp. A phage AP1 from A. pasteurianus NBRC 109446 was predicted as an active phage based on the genomic information, and actually had the ability to infect its phiAP1-cured strain. The attachment sites for phiAP1 were located in the $3^{\prime}$-end region of the $t R N A^{\text {ser }}$ gene. We also developed a chromosome-integrative vector, p2096int, based on the integrase function of phiAP1, and it was successfully integrated into the attachment site of the phiAP1-cured strain, which may be used as a valuable tool for the genetic engineering. Overall, this study showed the distribution of mitomycin $\mathrm{C}$-inducible temperate phages in $\mathrm{AAB}$, and identified the active temperate phage of $A$. pasteurianus.

Acetic acid bacteria (AAB) are Gram-negative and obligate aerobic bacteria belonging to the order Rhodospirillales of the class alphaproteobacteria ${ }^{1-3}$. AAB are frequently isolated from sugar-containing or acidic materials such as fermented food, beverages, animal organs, fruits, flowers, insect guts, and soil ${ }^{1-3}$. The AAB strains belonging to the Acetobacter, Gluconobacter, and Komagataeibacter genera have been mainly used to brew vinegar and produce functional molecules ${ }^{1}$. A. pasteurianus is used to brew cereal vinegars by liquid-state or solid-state fermentation in Asia countries such as Japan and China. One of the remarkable abilities of AAB is the oxidization of ethanol, sugar, or sugar alcohol, under the presence of oxygen, to organic acids such as acetic acid and gluconic acid ${ }^{\text {. }}$. This specific ability has been used for industrial fermentation of vinegar, a highly important organic acid in the food industry that contributing to food, bioengineering and medical fields.

Bacteriophages (phages) are bacteria-infective viruses with great diversity and are the most abundant biological entities ${ }^{4}$; they play an important role in bacterial ecology and evolution, including gene reservoir and horizontal transfer of genetic materials ${ }^{5-7}$. Phages are divided into four life cycles including the lytic cycle, the lysogenic cycle, pseudolysogeny, and chronic infection or shedding ${ }^{8}$. Lytic (also called virulent) phages amplify their genome after infection, and lyse the host cells to infect other healthy host cells. Temperate (also called lysogenic) phages have two modes: prophages integrate into host chromosome and propagate with host genome until the host is active, while prophages are induced (transfer to lytic phase) when physiological and chemical stress are generated by the treatment of UV irradiation, mytomycin C (MMC), and reactive oxygen species (ROS) as a DNA-damaging reagent ${ }^{9}$. The temperate phage-derived integrase gene encodes a member of the tyrosine or serine family recombinase catalyzing a site-specific recombination between two specific sites: the attachment sites of bacterial host $(a t t B)$ and phage $(a t t P)^{10}$. The lytic and temperate phages comprise a majority of the life cycle of phages.

Morphology and genome structures of virulent and temperate phages have been well studied in industrial bacteria, while a few studies on AABs phage have been reported as described below. As virulent phages of $A A B$, phages GW6210 and JW2040 isolated from rotten apples showed virulence to Gluconobacter oxydans ATCC 621 and G. oxydans VP1 $204 \mathrm{JW}^{11}$, respectively. As temperate phages, phage Acm 1 from Acetobacter methanolicus MB5814 $4^{12}$, phage A-1 from G. oxydans strain $\mathrm{E}^{13}$, and more recently, a novel tectivirus phage GC1 infecting $G$. cerinus associated with wine-making ${ }^{14}$ were reported. To the best of our knowledge, there have been no reports on phages associated with a major vinegar-producing A. pasteurianus and a cellulose-producing K. xylinum.

${ }^{1}$ Life Science Research Center, College of Bioresource Sciences, Nihon University, Kanagawa, Japan. ${ }^{2}$ Mizkan Holdings Co., Ltd, Aichi, Japan. ${ }^{\circledR}$ email: takano.hideaki@nihon-u.ac.jp 
Phage-like particles in vinegar fermentation have been experimentally observed in the culture broth when fermentation is halted (our unpublished observation). However, little has been characterized on virulent and temperate phages of AABs as described above. Here, we performed a comprehensive investigation of AAB temperate phages induced by MMC treatment. Understanding the phage diversity and genomic structures will aid in improving biotechnological processes for preventing the fermentation from halting. In this study, we detected 15 myoviridae-type temperate phages among a total of $177 \mathrm{AAB}$ strains, and we determined the complete genome sequences of the six temperate phages produced from Acetobacter and Komagataibacter spp. We also report that a phage AP1 (designated as phiAP1) from A. pasteurianus NBRC 109446 has the ability to infect its phiAP1cured strain. Finally, we have developed a chromosome-integrative vector based on the function of phiAP1 as a valuable tool for genetic engineering.

\section{Results}

Distribution of phage-like elements in the genomes of acetic acid bacteria (AAB). Viral DNAs of temperate phages are ubiquitously found in the genomes of phylogenetically diverse Gram-positive and Gram-negative bacteria; however, little is known about AAB temperate phages compared to other industrially used bacteria. We first searched for prophage-like elements using a web-based tool PHAge Search Tool (PHAST 2016.12.23 Updated version; http://phast.wishartlab.com/ $/)^{15}$ against 22 whole genome sequences derived from the genera Acetobacter, Gluconobacter, Gluconacetobacter, and Komagataeibacter. As listed in Table S1, the harbor of phage-like elements in these genomes were predicted in all of the genome-sequenced AABs, and a total of 94 prophage-like elements were found in the $22 \mathrm{AAB}$ strains. The average number of temperate phages per acetic acid bacterium is 4.3 , which is higher than that of other bacteria, $2.6^{16}$, indicating that AAB strains potentially have a larger number of prophage-like elements than other bacteria.

The PHAST-based prediction grouped the phages into three types: intact (active phage), incomplete (degenerate phage), and questionable (where it was impossible to predict whether the phage is active or degenerate). The temperate phages predicted as active were found in 12 of the $22 \mathrm{AAB}$ strains. To the best of our knowledge, there are no previous reports of temperate phages derived from A. pasteurianus and K. xylinum; however, the PHAST analysis suggests that temperate prophage-like elements might be widespread in these bacterial groups. These results strongly suggest the existence of active temperate phages in these industrial microorganisms.

Screening of lysogens in AAB. To survey the distribution of active temperate phages existing as prophage within the $\mathrm{AAB}$ genomes, we first screened lysogenic $\mathrm{AAB}$ strains mainly belonging to the Acetobacter, Gluconobacter, Gluconoacetobacter, and Komagataeibacter genera, which were obtained from publically or commercially available culture collections such as JCM, NBRC, IAM, ATCC and DSMZ. A total of 177 AAB strains (Table S2) were grown on YPG solid media in the absence or presence of low concentration of mitomycin C (MMC), which is frequently used as an inducer agent for temperate phage due to its inhibitory activity of DNA synthesis. As listed in Table S3, 27 out of 177 strains showed a MMC-sensitive growth profile, in which the growth inhibition by MMC might be due to host cells lysis followed by phage induction. This growth inhibition was also confirmed when strains were grown in MMC-containing liquid shaking culture. The above 27 strains were composed of 10 strains of Acetobacter spp, seven strains of Komagataeibacter spp, one strain of Gluconoacetobacter sp, six strains of Gluconobacter spp, and one strain each of Frateuria sp., Acidomonas methanolica ATCC 43582, and Ameyamaea chiangmaiensis NBRC 103196. These results imply that approximately $15.2 \%$ of the AAB strains retain a MMC-inducible temperate phage.

Transmission electron microscopy (TEM) analyses of phage particles. To confirm whether the above 27 strains actually produced phage particles in response to MMC, we performed TEM analyses using the phage-containing fractions, which were prepared by concentrating the supernatant from the culture broth of the MMC-treated lysogens (See Materials and Methods). We successfully observed phage particles in 15 out of 27 lysogens with TEM analyses (Table 1, Fig. 1, and Fig. S1). The number of the lysogens observed was four for Acetobacter strains, six for Komagataeibacter, three for Gluconobacter, one for Acidomonas, and one for Ameyamaea. TEM analyses also revealed features of their morphology; the observed phage particles retained their heads and contractile tails. Based on morphology, all of the phages were classified into the myoviridae family with icosahedral capsids $41 \pm 5 \mathrm{~nm}$ in diameter, and tails $156 \pm 6 \mathrm{~nm}$ in length (Table 1). Overall, these results suggest that a large proportion $(8.4 \%)$ of $\mathrm{AAB}$ are capable of producing myoviridae-type temperate phages. The MMC induction of phage particles indicates that the trigger is linked to the SOS response of the bacterial host. The growth inhibition observed in other MMC-sensitive strains might due to the generation of degenerate phage.

Complete genome sequences of AAB temperate phages. To reveal the genome structures and gene composition of the temperate phages observed in the TEM analyses, we selected one or two strains from each genus/species among the lysogens isolated from the vinegar fermenter. We then sequenced the whole genomes of six temperate phages using next generation sequencing technique. Genomic DNAs were purified from the phage-fraction prepared by concentrating the supernatant of the MMC-treated AABs (See Materials and Methods). The complete genome sequences without any gaps were determined in the six phages, and each gene product was annotated. Table S4 shows the annotation of each phage genome. In each strain, one phage genome was detected in the genome-sequencing analysis. As shown in Fig. $2 \mathrm{~A}$, we designated the six phages as phiAP1 from A. pasteurianus NBRC 109446, phiAO1 from A. orleanensis NBRC 3170, phiAX1 from Acetobacter sp. ATCC 21760, phiKX1 from Komagataeibacter xylinus NBRC 13772, phiKX2 from K. xylinus NBRC 13773, and phiKM1 from K. maltaceti NBRC 14815. 


\begin{tabular}{|c|c|c|c|c|c|}
\hline Host strain & Isolation source $\mathrm{e}^{\mathrm{a}}$ & Head $^{b}$ & Tail $^{\text {b }}$ & $n^{c}$ & Tail-fiber $^{d}$ \\
\hline \multicolumn{6}{|l|}{ Acetobacter } \\
\hline A. orleanensis NBRC 3170 & Manufacture of vinegar & $43.1(0.0)$ & $46.6(1.4)$ & 3 & - \\
\hline A. orleanensis ATCC 6438 & Manufacture of vinegar & $45.0(1.3)$ & $83.3(13.6)$ & 3 & - \\
\hline A. pasteurianus NBRC 109446 & Vinegar fermentor & $68.8(0.0)$ & $86.1(3.9)$ & 3 & - \\
\hline Acetobacter sp. ATCC 21760 & - & $44.8(0.8)$ & $135.8(9.5)$ & 3 & - \\
\hline \multicolumn{6}{|l|}{ Komagataeibacter } \\
\hline K. maltaceti NBRC 14815 & Malt vinegar brewery acetifiers & $47.4(1.3)$ & $104.6(6.1)$ & 3 & - \\
\hline K. xylinus NBRC 13693 & - & 52.1 & 60.3 & 1 & - \\
\hline K. xylinus NBRC 13772 & Film in fermentor of vinegar & $65.6(1.5)$ & $98.9(3.0)$ & 3 & - \\
\hline K. xylinus NBRC 13773 & Film in fermentor of vinegar & $55.0(4.1)$ & $95.0(10.8)$ & 3 & - \\
\hline K. xylinus ATCC 14851 & Vinegar brew & 60.2 & 82.6 & 1 & - \\
\hline K. xylinus ATCC 53264 & - & $62.1(0.0)$ & $56.0(0.9)$ & 2 & - \\
\hline \multicolumn{6}{|l|}{ Gluconobacter } \\
\hline G. cerinus IAM 1832 & Apple & $50.0(1.5)$ & $94.6(1.5)$ & 3 & - \\
\hline G. cerinus ATCC 23775 & Baker's yeast & $54.5(0.0)$ & $83.3(7.7)$ & 3 & - \\
\hline G. frateurii IAM 1815 & - & $59.2(2.2)$ & $71.8(2.2)$ & 3 & - \\
\hline \multicolumn{6}{|l|}{ Others } \\
\hline Acidomonas methanolica ATCC 43582 & Ditch sludge & $44.4(0.0)$ & $75.3(1.7)$ & 3 & - \\
\hline Ameyamaea chiangmaiensis NBRC 103196 & Flower of red ginger, Alpinia purpurea & $67.5(0.0)$ & $15.0(0.0)$ & 2 & - \\
\hline
\end{tabular}

Table 1. Morphological characteristics of AAB temperate phages. ${ }^{a}$ Dashes indicate unknown; ${ }^{b}$ Values are Mean (SD) of phage size $(\mathrm{nm}){ }^{c} \mathrm{n}$ is measurement number of phage particle; ${ }^{\mathrm{d}}$ Dashes indicate not detected.

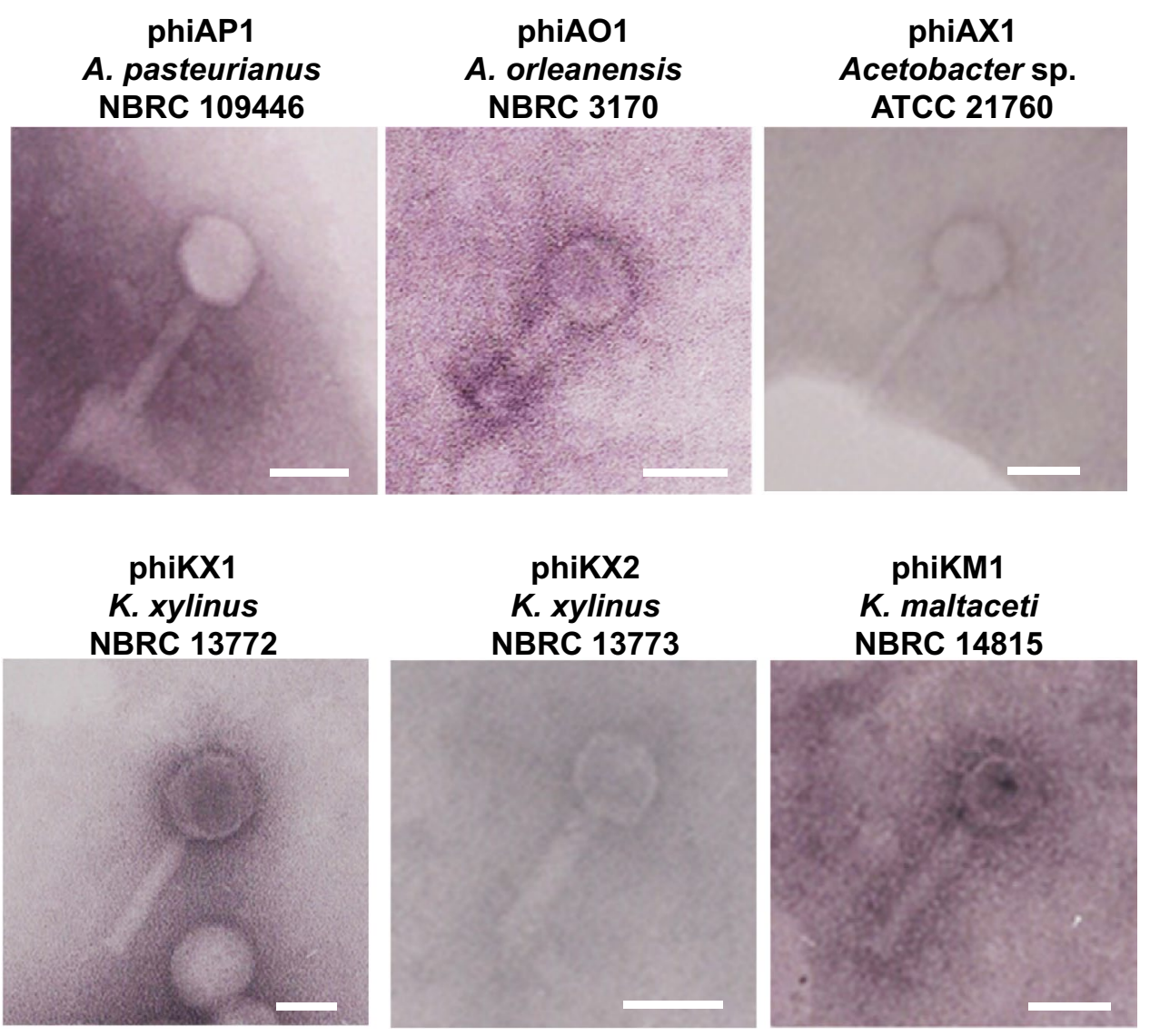

Figure 1. TEM photographs of temperate phages produced by acetic acid bacteria. Phage particles of acetic acid bacteria $(\mathrm{AAB})$ were photographed with TEM. Fractions were prepared from AAB grown in YPG-1\% glucose medium containing mitomycin $\mathrm{C}$ (MMC) with shaking culture for $48 \mathrm{~h}$. Bars, $50 \mathrm{~nm}$. 
A

phiAP1 of $A$. pasteurianus NBRC 109446 (42,805 bp, Circular)

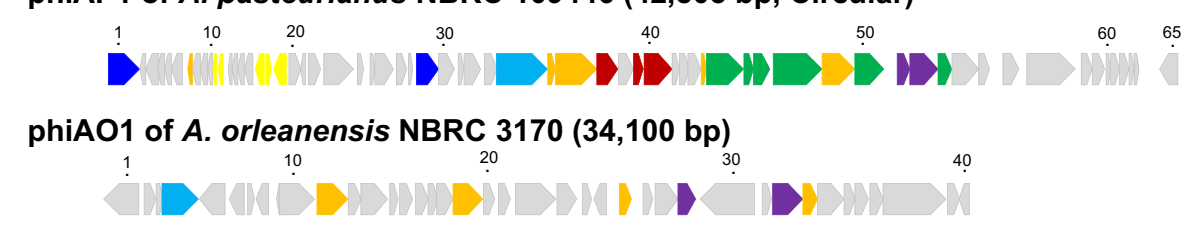

phiAX1 of Acetobacter sp. ATCC 21760 (50,652 bp)

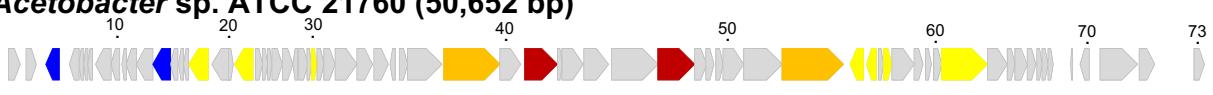

phiKX1 of $K$. xylinus NBRC 13772 (53,192 bp , Circular)

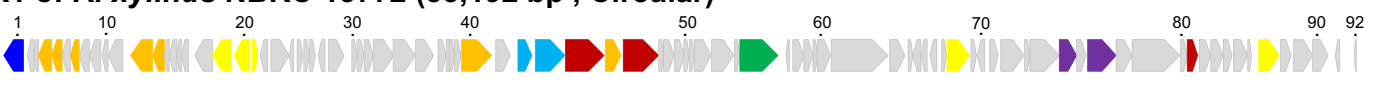

phiKX2 of K. xylinus NBRC 13773 (53,798 bp)

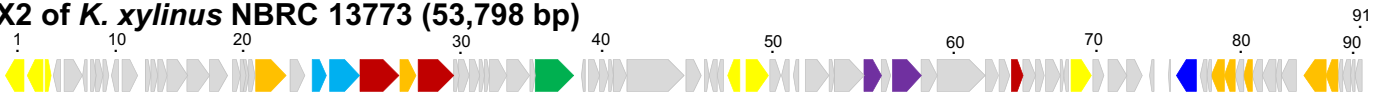

phiKM1 of $K$. maltaceti NBRC 14815 (51,977 bp)

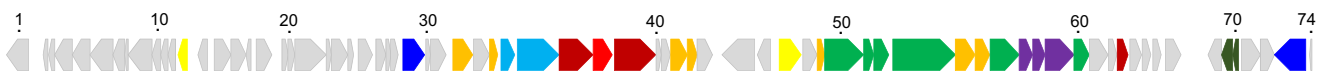

B

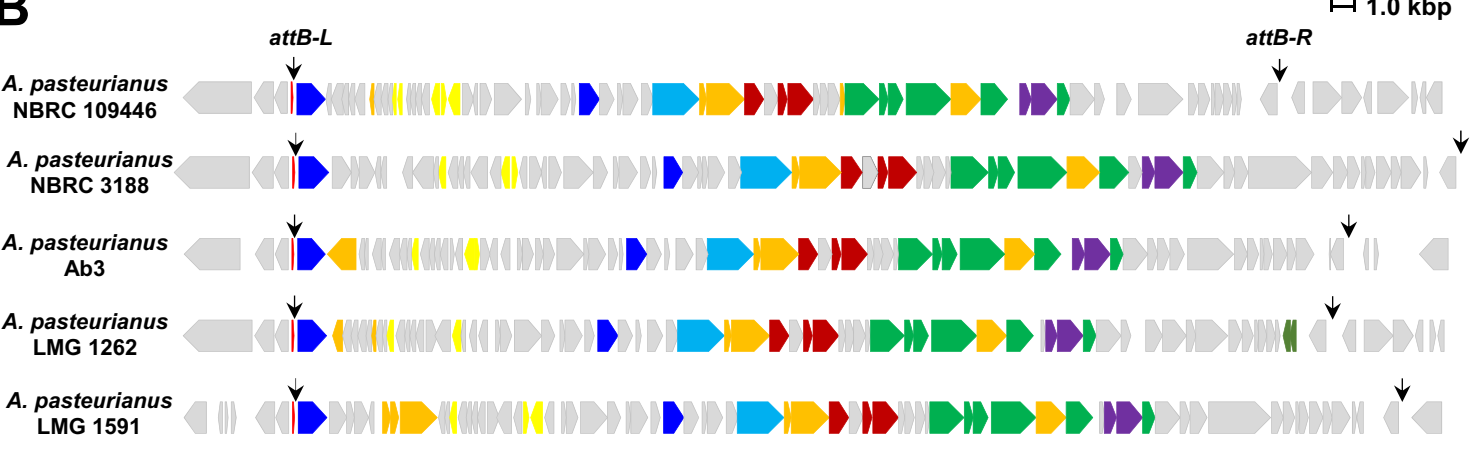

. pasteurian

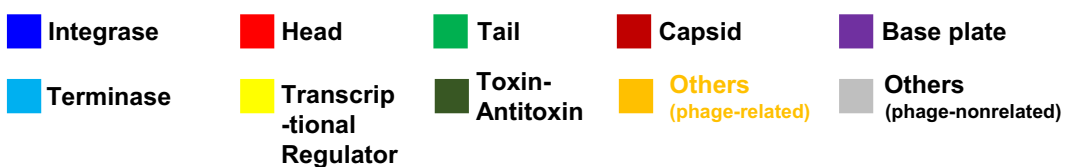

Figure 2. Schematic representation of temperate phage genomes in AAB. (A) Genome structure and gene organization of the temperate phages determined in this study. Number above arrow represent ORF number in each temperate phage (annotations for each gene product are shown in Table S4). Numbers in parentheses indicate the genome size and structure of the phages. (B) Distribution of phiAP1-like prophage elements in the genomes of $A$. pasteurianus strains. Downward arrowheads indicate the putative attB- $L$ and attB-R sites.

Figure 2A shows the predicted gene organization based on phage genome information. Genome sizes range from 34,100 to $53,798 \mathrm{bp}$. Circular genome were confirmed in phiAP1 and phiKX1. The molecular $\mathrm{G}+\mathrm{C}$ content of each genome is $52.4 \%$ for phiAP1, 61.4\% for phiAO1, $56.0 \%$ for phiAX1, $60.2 \%$ for phiKX1, $60.0 \%$ for phiKX2, and $62.2 \%$ for phiKM1 (Table S4). The molecular G + C contents of A. pasteurianus IFO 3283-01 are 50. 7\% in the chromosome and $55.8 \%$ in the six plasmids ${ }^{17}$, and that of K. xylinus E25 are $62.6 \%$ in the chromosome and $57.8 \%$ in the five plasmids ${ }^{18}$. The $\mathrm{G}+\mathrm{C}$ contents of the six phages lie in the between the values of chromosome and plasmids in both genera.

In each of the phage genomes, open reading frames (ORFs) were found: 65 in phiAP1, 40 in phiAO1, 72 in phiAX1, 91 in phiKX1, 90 in phiKX2, and 73 in phiKM1 (Fig. 2A and Table S4). Most genes are predicted to be composed of an operon structure, because the initiation codon and stop codon of translation are close or overlapped in the ORFs. 
Phage integrase recognizes two sites, a bacterial attachment site (attB) and a phage attachment site (attP), and catalyzes the site-specific recombination between $a t t B$ and $a t t P$ to generate the integrated state of phage flanked with $a t t B-L$ and $a t t B-R^{10}$. Most integrase are classified into two families, tyrosine and serine recombinase families ${ }^{10}$. Integrase genes were found in all prophage genomes studied except for that of $A$. orleanesis NBRC 3170 (Fig. 2A). These integrases are classified into tyrosine family integrases based on Pfam search. The existence of integrase in the phage genome suggests that these phages serve as a temperate type.

To examine the functionality of phages, their genomes were analyzed by PHAST. The two phages, phiAP1 and phiKM1, were predicted as active phages. In contrast, phiAO1, phiAX1, and phiKX1 were predicted as incomplete, indicating degenerate prophages; and phiKX2 was predicted as questionable. This implies that the three phages predicted as incomplete have an ability to assemble the phage body structure, but lack the infection to the host strain. In support of this prediction, phiAP1 and phiKM1 retains many phage-related genes in their genomes (Fig. 2A). In contrast, phiAO1 harbors only nine phage-related genes out of a total 40 genes. Thus, these data support that phiAP1 and phiKM1 serve as functional phages, which are able to infect AAB strains.

Distribution of phiAP1-like elements in Acetobacter spp. We searched the distribution of phiAP1like elements in AABs using publically available genomic information. Four strains of A. pasteurianus including NBRC 3188, Ab3, LMG 1262, and LMG 1591 retained a phiAP1-like prophage element in their genomes (Fig. 2B). In contrast, A. pasteurianus NBRC 3283 and 386B have not phiAP1-like element in their genomes. The composition and direction of the phage-related genes located in the center and right region of the genome are highly conserved among the four Acetobacter strains, while the composition of the small ORFs located in the flanking region of attB- $L$ are slightly different in each strain. We also found that putative attB- $L$ and $a t t B-R$ have a high similarity to each other among the four Acetobacer spp. as described below. PHAST analysis suggested that all of the above phiAP1-like phages are active, suggesting that they have the ability to infect Acetobacter spp. The high similarity of phiAP1-like elements among the five strains suggests that their origin is identical, and that they are spread by phage infection.

Construction of a phiAP1-cured strain in A. pasteurianus NBRC 109446. Among the phages observed with TEM analyses, phiAP1 is predicted as an active temperate phage, and phiAP1-like elements are found in other A. pasteurianus strains as described above. Among the detected temperate phages, our study focused on phiAP1 derived from A. pasteuianus NBRC 109446, because the host strain was isolated from a vinegar fermentation facility in Japan. To examine the ability of phiAP1 in infecting A. pasteurianus NBRC 109446, we first constructed a phiAP1-cured strain, due to the general presence of a system preventing multiple phage infection which protects their lysogenic host by superinfection exclusion and homo-immunity against infecting phages $^{19}$.

We analyzed approximately 1,200 colonies of MMC-treated A. pasteurianus NBRC 109446 using PCR analysis to confirm the loss of the phiAP1 prophage genome. Tween 80 was added to prevent phage reinfection by inhibiting phage adsorption ${ }^{20}$. As a result, we obtained a single phiAP1-cured strain among the ca. 1,200 colonies, which was designated as a C-27 strain. The inability of the C-27 strain to produce MMC-induced phages was confirmed with TEM analyses. This suggests that phiAP1 is the sole MMC-inducible phage in A. pasteurianus NBRC 109446 in the cultures condition used in this study.

Attachment sites recognized by phiAP1 Integrase. Nucleotide sequences of attB sites (attB- $L$ and $a t t B-R$ ) are generally similar to each other, and they are located in the neighborhood region of phage integrase gene and/or within host $t R N A$ gene ${ }^{21}$. We then searched attB sites for phiAP1, and found putative attB- $L$ (5'-CACCCCATCCGCCAACTATACTTC-3') and $a t t B-R$ (5'-CACCCCATCCGCCAACTATGCTTC- $\left.3^{\prime}\right)$ in the upstream region of the integrase gene (Fig. $2 \mathrm{~B}$ ) and around the $3^{\prime}$ region of $t R N A^{\text {ser }}$ gene (anticodon:TGA), respectively (Fig. 3A). Two attBs were $24 \mathrm{bp}$ in total length with a $1 \mathrm{bp}$ difference shown by underline. We also analyzed the $a t t B$ site of the phiAP1-cured region in the $\mathrm{C}-27$ strain, and assigned the following nucleotide sequences 5'-CACCCCATCCGCCAACTATGCTTC-3' (Fig. 3B) as an attB.

We then searched the phage attachment site, attP, in the phiAP1 genome. The predicted attP sequence had a $1 \mathrm{bp}$ difference to $a t t B$ - $L$ (Fig. $3 \mathrm{C}$ ). Based on the high nucleotide sequence similarity among attachment sites, it seems likely that Integrase encoded in phiAP1 catalyzes site-specific recombination between attB and attP to insert the phage genome into the $t R N A^{\text {ser }}$ gene in the host chromosome, and resulting in the generation of a prophage retaining $a t t B-L$ and $a t t B-R$ (Fig. 3C). The integration does not result in disruption of the $t R N A^{\text {ser }}$ gene (Fig. 3A,B).

Conservation of the phiAP1-like attB site in Acetobacter spp. To investigate the conservation of phiAP1-like elements, we analyzed the synteny of the $t R N A^{\text {ser }}$ locus in the genome-sequenced Acetobacter stains (Fig. 4). The $t R N A^{\text {ser }}$ and an elongation factor $\mathrm{P}$ genes are completely conserved in this genus. We found that phiAP1-like elements are located in the region adjacent to $t R N A^{\text {ser }}$ in the four $A$. pasteurianus strains including NBRC 3188, Ab3, LMG 1262, LMG 1591, and A. oryzifermentans SLV-7. These phiAP1-like elements were predicted by PHAST analysis to form active phage. This result indicates that phiAP1-like elements are distributed and located in the same locus or genomic region across the A. pasteurianus strains.

In order to predict att $B$ sites located in the above conserved region, we compared the nucleotide sequences of the $t R N A^{\text {ser }}$ gene and its 3'-flanking region (Fig. 5). The full length attB- $L$ and attB-R sequences of $A$. pasteurianus NBRC 109446 were only conserved in A. pasteurianus LMG 1262 strain with a 1 bp-substitution. Whereas the attB sites in the other four lysogenic strains (A. pasteurianus NBRC 3188, Ab3, LMG 1591, and A. ascendens SRCM 101447) were predicted as 5'-CACCCCATCCGCCA-3' with a length of $14 \mathrm{bp}$. The short attB- $L$ site with 
A

GCTGTTTCATAATTCGTCCCTGAAAGgAATGTCCATTAAGGATGGGGCTTGTACAAGCCTGACGCCCGA

AGAGCAAGTTTACTGCGTTTGATATATCGTGTTTTCTCCCGCTGGACAGGGTGGCCATATCCTGTTTAT $>$ tRNA ser

AAgGCGGCAGGTCCTCAGATGGATGAATCGAGGATGGGTGTCCGAGTGGTTTAAGGAACTGGTCTTGAA attB-L

AACCAGCGTGCGGGGAACCGTACCGTGGGTTCGAATCCCACCCCATCCGCCAАCTATACTTCCCTATAG

TTCCCAATTATTCCCAAAGCCCTTGCAAAACTGCCAGGTATAGGGTTTTTGTGTTCCCAATCGTTCTCG

TTTTATCCCATCCGTTCCCGCGCAAATGTGGGTAACGTGTGGGTAAGATTTTCAGGATGTGTGGGTAAC

GCAATCATGCTGACAGATTCCAAGGTCAAGACAGCTAAGGCAGCCGAGAAAGCCTATCGCCTTTCTGAT

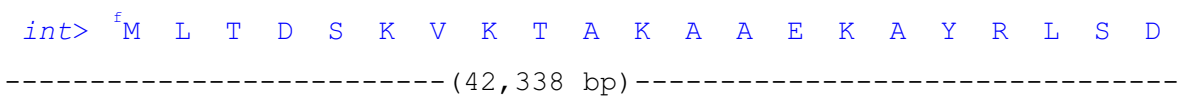

GTGATGGATGATGGAATCGTTTGGCATAACAAAATTCTGCTTCATGGAAGTGTTGGTTGGTGATCTGAA

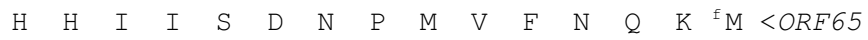

ATTTACCTTAATGCATATAGAAAGGCATTTAGGCATCACATACAAGCGCTCAAAATTTGTGGGTAATTC

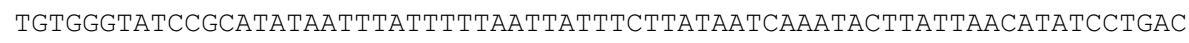
TGACACCCCATCCGCCAACTATGCTTCTCCAAAATCTTTAATATCTTTGTAAAATGGTTTGCTGGCTCC attB-R

\section{B}

$>$ tRNA $A^{\text {Ser }}$

GGATGGGTGTCCGAGTGGTTTAAGGAACTGGTCTTGAAAACCAGCGTGCGGGGAACCGTACCGTGGGTT CGAATCCСАССССАTCCGCCAACTATGCTTCTCCAАAATCTTTAATATCTTTGTAAAATGGTTTGCTGG CTCC

attB

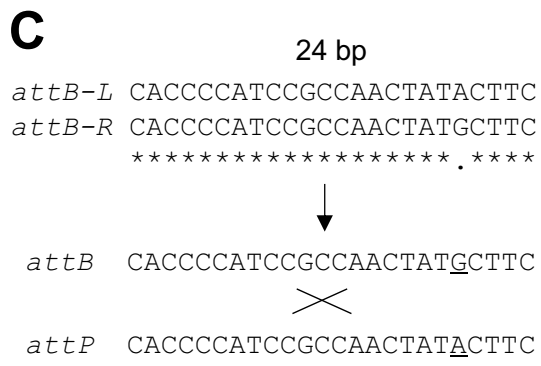

Figure 3. Nucleotide sequences of att sites in A. pasteurianus NBRC 109446 and phiAP1. (A) Bacterial host att sites $(a t t B-L$ and $a t t B-R)$ underlined are located in the genomic boundary region between the host and phiAP1. The $t R N A^{\text {ser }}$ gene is shown in red letters. (B) The attB site in the phiAP1-cured C-27 strain is underlined. The $t R N A^{\text {ser }}$ gene is shown in red letters. (C) Comparison of the nucleotide sequences of the att sites with a length of $24 \mathrm{bp}$.

$14 \mathrm{bp}$ length is also highly conserved in non-lysogenic strains (Fig. 5). This suggests that the short attB sites are also recognized by Integrase.

Infection of $\boldsymbol{A}$. pasteurianus C-27 strain by phiAP1. Our genome analysis implies that phiAP1 is able to infect the host cell and integrate its genome into the chromosome via the function of integrase. We then examined the infective ability of phiAP1 with its phiAP1-cured strain C-27. Unexpectedly, we were unable to observe any plaques on confluent lawns of the C-27 strain, despite testing the assay using various phage-fractions prepared from different culture conditions. This result suggests that phiAP1 rapidly and stably integrates into the host genome after infection, and it do not form plaques on host cell lawns by maintaining a stable lysogenic cycle. In order to enable selection of the phiAP1-infected cells, we constructed a modified phiAP1 that carries an ampicillin-resistance gene on its genome (designated as phiAP1-Amp). The phiAP1-Amp particles induced from a host GMS3 strain harboring phiAP1-Amp prophage were observed with TEM analyses (Fig. S2). We then spotted phiAP1-Amp-containing fractions onto the lawns of the C-27 strain, and screened for ampicillin-resistant C-27 strains after 2 days of co-incubation. The C-27 strain grown in the phage-spotted region was harvested with a spatula, and the cell suspended in sterile distilled water was inoculated onto YPG solid medium containing ampicillin. As shown in Fig. 6, the ampicillin-resistant C-27 strains were observed on YPG solid medium supplemented with ampicillin. The specific integration of phiAP1-Amp into the attB site was confirmed by PCR analysis. In contrast, no colonies were observed when phiAP1-Amp was co-incubated with the wild-type strain. 
A. pasteurianus NBRC 109446

A. pasteurianus NBRC 3188

A. pasteurianus Ab3

A. pasteurianus LMG 1262

A. pasteurianus LMG 1591

A. oryzifermentans SLV-7

A. pasteurianus 386B

A. pasteurianus IFO 3283

A. ascendens SRCM101447

A. pomorum BDGP5

Acetobacter sp. JWB

A. ascendens LMG 1590

A. tropicalis BDGP1

A. persici TMW2.1084

A. senegalensis

A. aceti

TMW2.1153
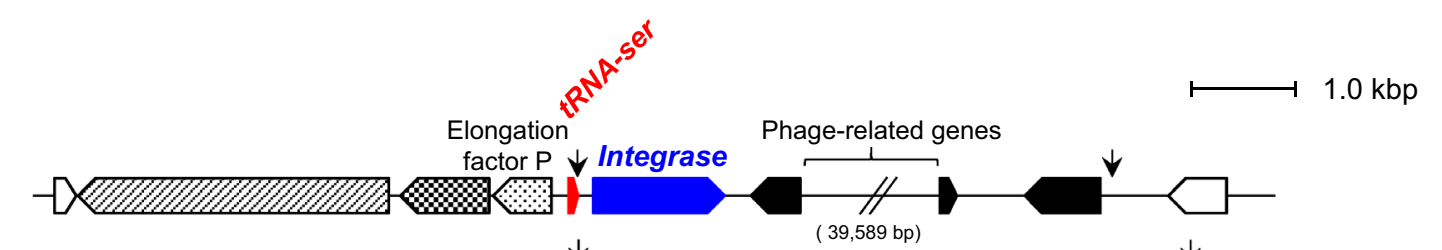

Elongation
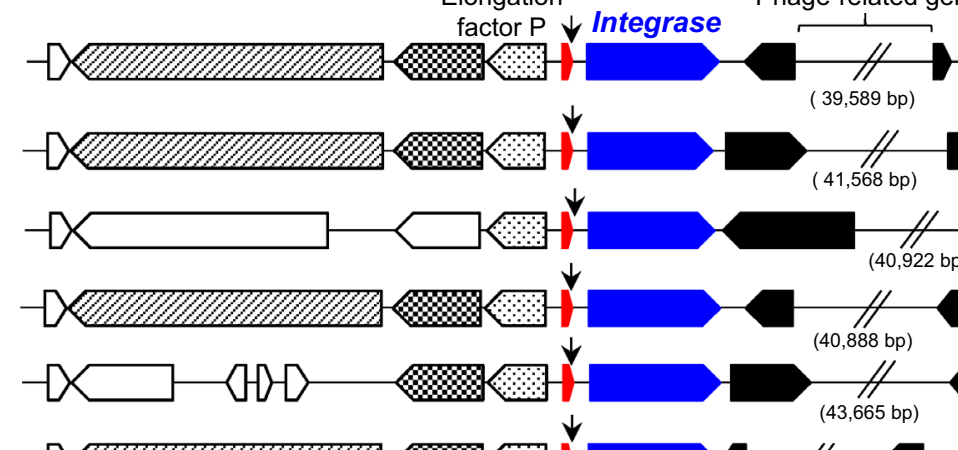

Phage-related genes
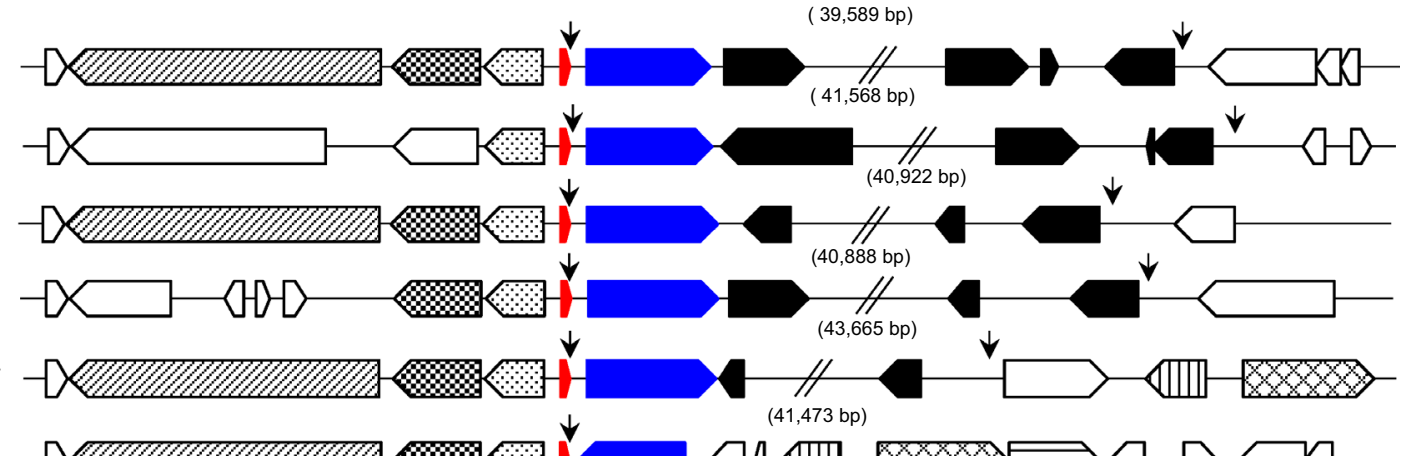
$(41,473 \mathrm{bp})$
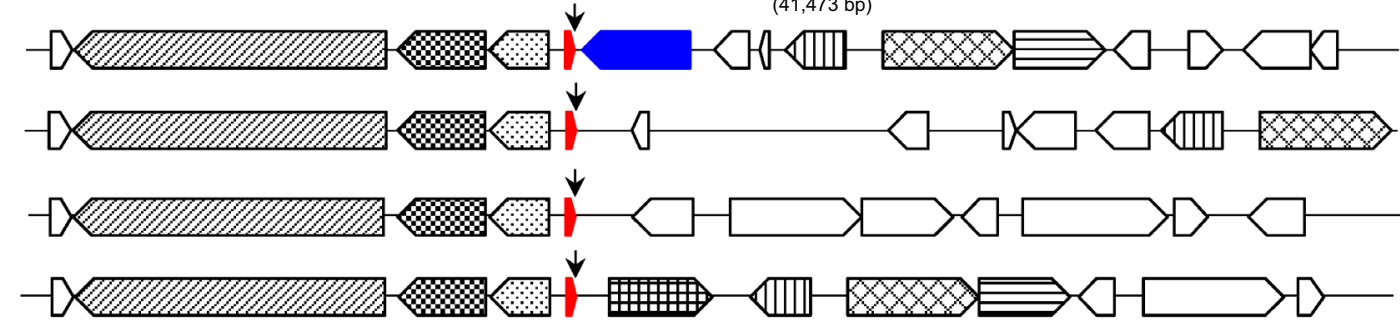

$\downarrow$
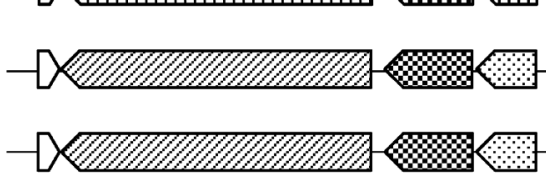

$\downarrow$
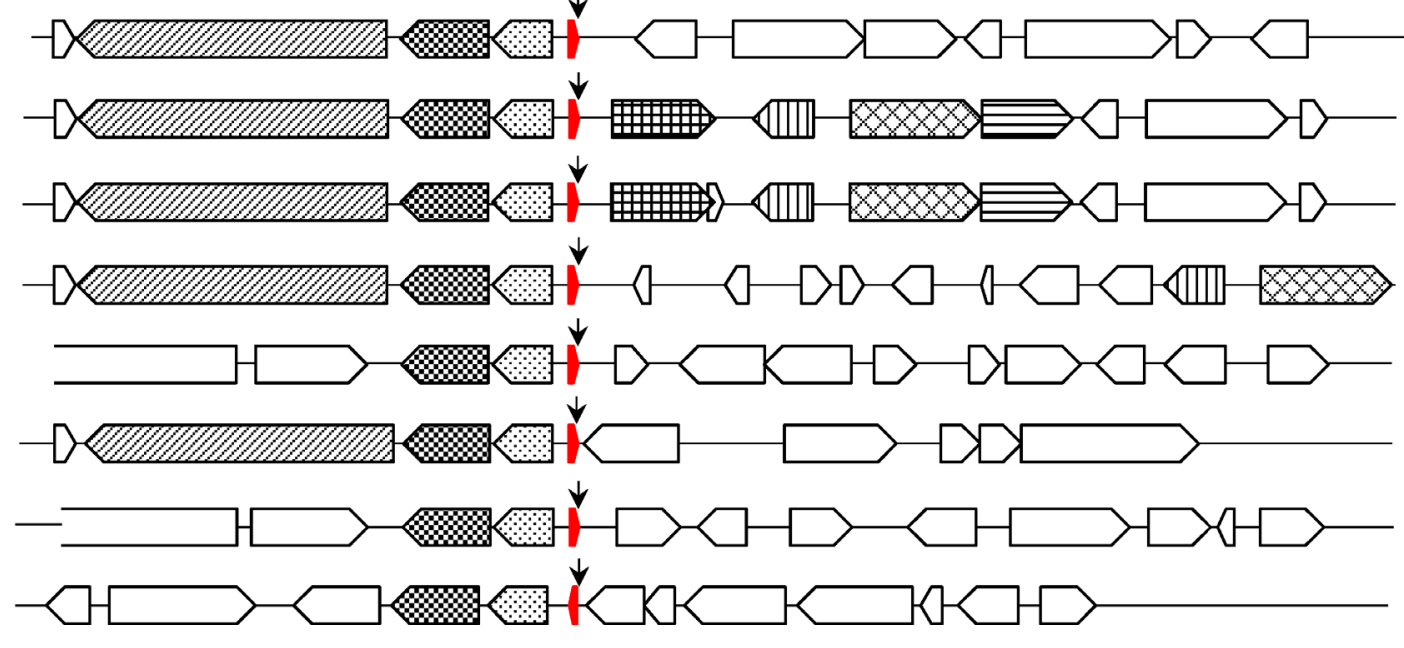

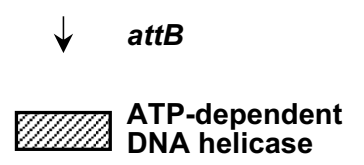

Q
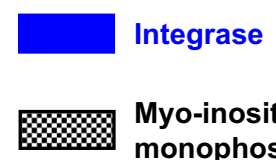

Myo-inositol-1(or 4)monophosphatase

cysE

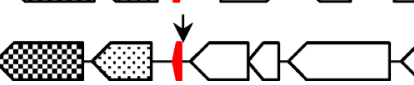

\section{K}
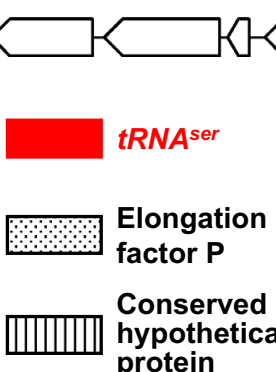

$t_{R N A}{ }^{\text {ser }}$

Prophage

region

Elongation

factor $P$

gale

Conserved

hypothetical protein

others

Figure 4. Gene organization of $t R N A^{\text {ser }}$ gene locus in Acetobacter spp. The gene compositions and arrangements located in the $t R N A^{\text {ser }}$ locus of Acetobacter spp. are shown. The upstream region of $t R N A^{\text {ser }}$ was highly conserved among the genus of Acetobacter. Arrowheads indicate the putative attB sites. Genes for $t R N A^{\text {ser }}$ and integrase are indicated by red and blue-colored arrowheads, respectively.

This result indicates that phiAP1 has the ability to specifically infect the cured host cell, and integrate into the genome.

Construction of a chromosome-integrative vector, p2096int, for A. pasteurianus NBRC 109446. Lysogeny is established by site-specific recombination between the temperate phage genome and bacterial chromosome, and this process is catalyzed by phage integrase. To evaluate the function of phiAP1 integrase and develop a useful molecular tool for $\mathrm{AAB}$ research, we constructed a vector capable of integrating into the chromosome (designated as p2096int). The general feature of a chromosome-integrative vector is specific and efficient integration into att $B$, and stable maintenance in the chromosome ${ }^{21}$ which enables the introduction of large gene clusters such as the secondary metabolite biosynthesis gene cluster. This type of vec- 


\begin{abstract}
$>$ tRNA ${ }^{\text {ser }}$
Anticodon

(TGA)

A. pasteurianus NBRC109446

A. pasteurianus NBRC3188

A. pasteurianus ATCC23754

A. pasteurianus Ab3

A. pasteurianus LMG1262

A. pasteurianus LMG1591

A. oryzifermentans SLV-7

A. pasteurianus $386 \mathrm{~B}$

A. pasteurianus IF03283

A. ascendens SRCM101447

A. ascendens LMG1590

A. pomorum BDGP5

A. sp. JWB

A. senegalensis

A. tropicalis BDGP1

A. persici TMW2. 1084

GGATGGGTGTCCGAGTGGTTTAAGGAACTGGTCTTGAAAACCAGCGTGCG GGATGGGTGTCCGAGTGGTTTAAGGAACTGGTCTTGAAAACCAGCGTGCG GGATGGGTGTCCGAGTGGTTTAAGGAACTGGTCTTGAAAACCAACGTGCG GGATGGGTGTCCGAGTGGTTTAAGGAACTGGTCTTGAAAACCAGCGTACG GGATGGGTGTCCGAGTGGTTTAAGGAACTGGTCTTGAAAACCAGCGTGCG GGATGGGTGTCCGAGTGGTTTAAGGAACTGGTCTTGAAAACCAGCGTGCG GGATGGGTGTCCGAGTGGTTTAAGGAACTGGTCTTGAAAACCAGCGTGCG GGATGGGTGTCCGAGTGGTTTAAGGAACTGGTCTTGAAAACCAGCGTGCG GGATGGGTGTCCGAGTGGTTTAAGGAACTGGTCTTGAAAACCAGCGTGCG GGATGGGTGTCCGAGTGGTTTAAGGAACTGGTCTTGAAAACCAGCGTGCG GGATGGGTGTCCGAGTGGTTTAAGGAACTGGTCTTGAAAACCAGCGTGCG GGATGGGTGTCCGAGTGGTTTAAGGAACTGGTCTTGAAAACCAGCG TGCG GGATGGGTGTCCGAGTGGTTTAAGGAATGGTCTTGAAAACCAGCGTGCG GGAAGGGTGGCCGAGTGGTTGAAGGCTCCAGTCTTGAAAACTGGCGTGCG GGAAGGGTGGCCGAGTGGTTGAAGGCTCCAGTCTTGAAAACTGGCGTGCG GGAAGGGTGGCCGAGTGGTTGAAGGCTCCAGTCTTGAAAACTGGCG TGCG

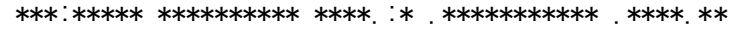

attB- $L$ or attB

attB- $R$

A. pasteurianus NBRC109446

A. pasteurianus NBRC3188

A. pasteurianus ATCC23754

A. pasteurianus Ab3

A. pasteurianus LMG1262

A. pasteurianus LMG1591

A. oryzifermentans SLV-7

A. pasteurianus $386 \mathrm{~B}$

A. pasteurianus IF03283

A. ascendens SRCM101447

A. ascendens LMG1590

A. pomorum BDGP5

A. sp. JWB

A. senegalensis

A. tropicalis BDGP1

A. persici TMW2. 1084
GGGAACCGTACCGTGGGTTCGAATCCCACCCCATCCGCCAACTATACTTC /CACCCCATCCGCCAACTATGCTTC GGGAACCGTACCGTGGGTTCGAATCCCACCCCATCCGCCAACTATACTTC /CACCCCATCCGCCA GGGAACCGTACCGTGGGTTCGAATCCCACCCCATCCGCCAAATTACTTTG / N. D GGGAACCGTACCGTGGGTTCGAATCCCACCCCATCCGCCAACTATACTTC /CACCCCATCCGCCA GGGAACCGTACCGTGGGTTCGAATCCCACCCCATCCGCCAACTATACTTC /CACCCCATCCGCCAACTATACTTC GGGAACCGTACCGTGGGTTCGAATCCCACCCCATCCGCCAACTATACTTC /CACCCCATCCGCCA GGGAACCGTACCGTGGGTTCGAATCCCACCCCATCCGCCAACCACGCTTC /CACCCCATCCGCCA GGGAACCGTACCGTGGGTTCGAATCCCACCCCATCCGCCAGCACTTT--- / N. D GGGAACCGTACCGTGGGTTCGAATCCCACCCCATCCGCCAAATTATTCTG / N. D GGGAACCGTACCGTGGGTTCGAATCCCACCCCATCCGCCAACTATACTTC / N. D GGGAACCGTACCGTGGGTTCGAATCCCACCCCATCCGCCAAATTATTCTG / N. D GGGAACCGTACCGTGGGTTCGAATCCCACCCCATCCGCCATCAAAAGAAA / N. D GGTAACCGTACCGTGGGTTCGAATCCCACCCCTTCCGCCATCAGGGT--- / N. D GGTAACCGTACCGTGGGTTCGAATCCCACCCCTTCCGCCATCAGGTGATA / N. D GGTAACCGTACCGTGGGTTCGAATCCCACCCCTTCCGCCATCAGATTGTA / N. D ** *********************************:*******. GGGAACCGTACCGTGGGTTCGAATCCCACCCCATCCGCCATCAAAAGAAA / N. D

Figure 5. Multiple alignments of attB sites in Acetobacter spp. Nucleotide sequences of the $t R N A^{\text {ser }}$ gene and its 3' flanking region were compared with CLUSTAL W (see Materials and Methods). Anticodon (TGA) was located in the position 35 to $37 \mathrm{bp}$. The coding sequences of the $t R N A^{\text {ser }}$ gene are shown by the line on top of the alignment. Putative $a t t B-L$ and $a t t B-R$ are shown in red text. N.D., not detected. Asterisks, colons, and periods are indicative of completely, highly, and semi-conserved nucleotide sequences, respectively.

w/o

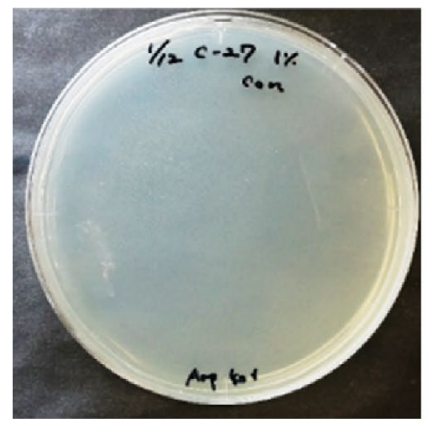

+ phiAP1

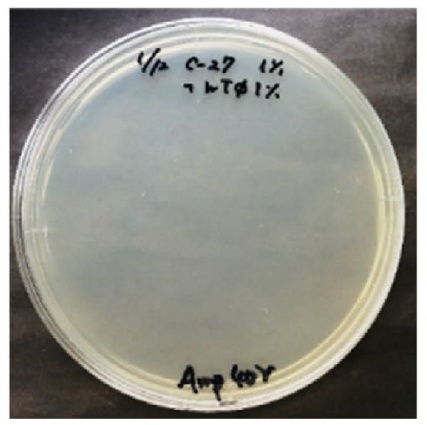

+ phiAP1-Amp

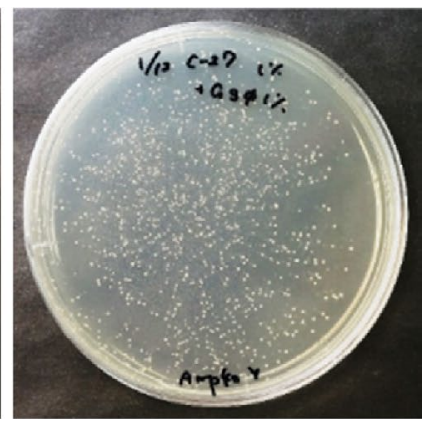

Figure 6. Infective ability of phiAP1 to the A. pasteurianus C-27 strain. The phiAP1 and phiAP1-Ampcontaining fractions were prepared from A. pasteurianus NBRC 109446 wild-type and GMS3 strains cultured with YPG-1\% glucose containing MMC. As a recipient cell, the C-27 strain grown in YPG-1\% glucose was used. The phage-infected C-27 strains were selected on YPG-1\% glucose containing ampicillin at $30{ }^{\circ} \mathrm{C}$ for 3 days. 


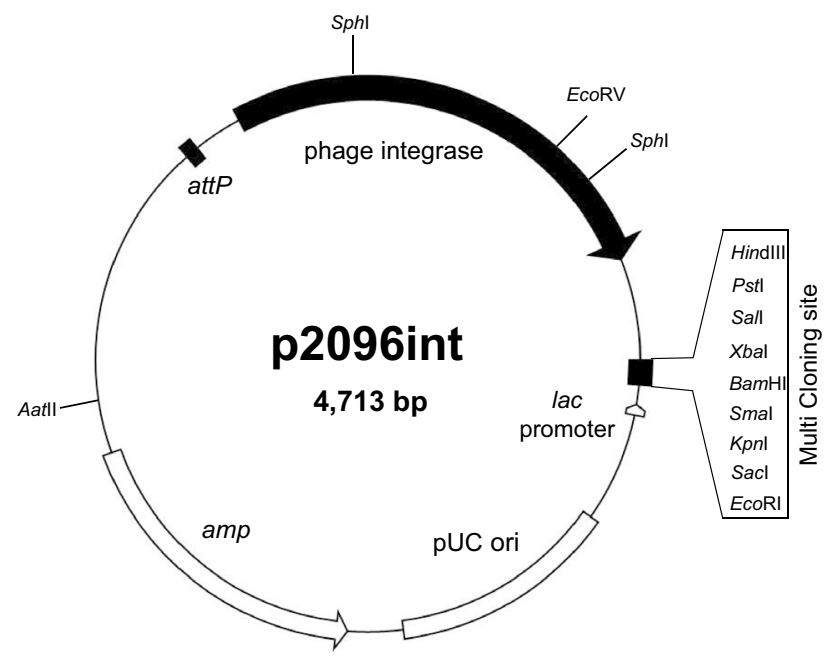

Figure 7. Plasmid map of a chromosome-integrative vector p2096int. p2096int vector contains attP, the integrase gene, multiple cloning sites (MCS), lac promoter, and an ampicillin-resistance gene. The vector is a pUC-based multicopy number plasmid propagated in E. coli, while it is a chromosome-integrative vector in the A. pasteurianus C-27 strain.

\begin{tabular}{|l|l|}
\hline Host strains & Transformants/ $\mathbf{\mu g}$ DNA \\
\hline A. pasteurianus NBRC 109446 & 0 \\
\hline A. pasteurianus C-27 strain & $6.1 \times 10^{3}$ \\
\hline A. pasteurianus NBRC 3188 & $2.4 \times 10^{2}$ \\
\hline A. pasteurianus ATCC 23754 & $1.1 \times 10^{3}$ \\
\hline
\end{tabular}

Table 2. Transformation efficiency with p2096int vector.

tor is also frequently used for genetic complementation experiments, because it's copy number is identical with the chromosome.

As shown in Fig. 7, we designed the components of an E. coli-Acetobacter shuttle vector p2096int carrying the integrase gene, attP, pMB1ori (pUC19-based non-replicative in Acetobacter), ampicillin-resistance gene, and multiple cloning site (MCS), and lacZalpha for blue-white selection. The introduction of p2096int into the phiAP1-cured C-27 strain led to formation of a large number of ampicillin-resistance colonies at $6.1 \times 10^{3} \mathrm{cfu}$ (transformants with $1 \mu \mathrm{g}$ DNA) (Table 2). The occurrence of the site-specific recombination between the attP and $a t t B$ site was confirmed by PCR analysis. On the other hand, no colony was obtained when p2096int was introduced into the A. pasteurianus NBRC 109446 wild-type strain, which suggests the existence of a mechanism preventing multiple phage infection by repressing integrase gene expression from p2096int. These results indicate that phiAP1 integrase is functionally active in A. pasteurianus, and p2096int is used as a site-specific chromosome-integrative vector.

To examine the host range of p2096int, we introduced this vector into total 38 strains of Acetobacter spp, 10 strains of Komagataeibacter spp, three strains of Gluconacetobacter spp, and two strains of Gluconobacter spp (Table S2). Two strains of $A$. pasteurianus were found to be recipients at a frequency of $2.4 \times 10^{2}$ cfu (transformants with $1 \mu \mathrm{g}$ DNA) in A. pasteurianus NBRC 3188, and $1.1 \times 10^{3} \mathrm{cfu}$ in A. pasteurianus subsp. pasteurianus ATCC 23754 (Table 2).

We determined the p2096int-integrated site in the two p2096int-accepted strains. In A. pasteurianus NBRC 3188 , p2096int was integrated at the attB- $L$ of the phiAP1-like element (Fig. 4), and the nucleotide sequences and length of attB- $L$ were completely identical with that of $A$. pasteurianus NBRC 109446 (Fig. 5). In A. pasteurianus ATCC 23754, p2096int was integrated at the 3' region of $t R N A^{\text {ser }}$ (Fig. 5), and the nucleotide sequences was partially identical with that of $A$. pasteurianus NBRC 109446. In this strain, phiAP1-like element was not found in the intergenic region between Elongation factor $\mathrm{P}$ and the upstream gene, which suggests that this strain does not retain phiAP1-like element in this genomic region.

We also analyzed the attP site of p2096int vector to determine the essential region for integration. The derivatives of p2096int vector were constructed, which retain the truncated attP site (Table 3). The CFU of p2096int-M1 vector retaining $7 \mathrm{bp}$ truncated $a t t P$ was apparently low compared with that of the parental p2096int. The CFU of p2096int-M2 and M3 was more low than that of p2096int-M1. This result suggests that efficient transformation with p2096int requires the $24 \mathrm{bp}$ attP with a full length. 


\begin{tabular}{|c|c|c|}
\hline Name of $a t t B$ site & Nucleotide sequence of $a t t B$ site $\left(5^{\prime}-3^{\prime}\right)^{a}$ & Transformants/ $\mu \mathrm{g}$ DNA \\
\hline p2096int & ATCCTGACTGACACCCCATCCGCCAACTATACTTCCCTATAGTTCC & $4.76 \times 10^{3}$ \\
\hline p2096int M-1 & ATCCTGACTGACACCCCATCCGCCAACTTCCCTATAGTTCCAATTA & $1.88 \times 10^{2}$ \\
\hline p2096int M-2 & ATCCTGACTGACACCCCATCCGCCATTCCCTATAGTTCCCAATTAT & $12.8 \times 10$ \\
\hline p2096int M-3 & 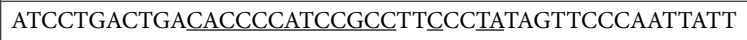 & $5.1 \times 10$ \\
\hline
\end{tabular}

Table 3. Transformation efficiency with p2096int vector retaining truncated $a t t B$ site. ${ }^{a}$ Nucleotide sequences of $a t t B$ were shown by underline.

\section{Discussion}

Our study showed that MMC-inducible temperate phages are widely distributed in the bacterial group of AAB used for industrial vinegar fermentation. The phage particles observed by TEM analyses were classified into myoviride-type, and the six of the genome structures were determined. Our study also revealed the presence of an infectious temperate phage, phiAP1, derived from A. pasteurianus. The phiAP1-like elements were highly conserved in the genomes of $A$. pasteurianus strains, suggesting that phiAP1 is a major prophage in this species. Our comparative analysis identified the att sites required for site-specific integration of the phage genome into the host chromosome catalyzed by phiAP1 integrase. We also applied the integrase gene to development of a chromosome-integrative vector p2096int, which was efficiently and specifically integrated into three A. pasteurianus strains. This study provides fundamental information related to and application of temperate phages in AABs.

Our wide investigation of temperate phages derived from a total of $177 \mathrm{AAB}$ strains showed that about $7.4 \%$ of AABs possess MMC-inducible temperate phages within their genomes. The requirement of MMC suggests that phage induction is linked to the SOS response of the host responsible for DNA repair. On the other hand, PHAST analysis suggests that $55 \%$ of AAB strains retain at least one of temperate phages in their genome. These suggest that SOS response of the host responsible for DNA repair, induced by physiological and chemical mutagens such as UV, MMC, and reactive oxygen species ${ }^{9}$, is a major pathway for phage induction. The large difference in the number between our analysis and genomic information also suggests that phage induction is required other stress generating in the acetic acid fermenter or AAB-dwelling environment. As far as we know, stress treatments other than MMC for inducing phage have not been found in this group of bacteria. Further study on stress for this group of bacteria generating in the fermenter or its actual dwelling environment will be to understanding the interaction of prophage and environments, and which is useful for the stable fermentation.

Morphological analyses using TEM supports that all of the AAB phages detected in this study belong to the myoviride type, which are found in a wide range of bacteria and archaea. Myoviridae type phages are nonenveloped with a head-tail, and their genome is a linear, double-stranded DNA, around 33 to $244 \mathrm{~kb}$ in length (https://viralzone.expasy.org/). Myoviridae type phages are generally lytic type, but temperate types have also been identified (https://viralzone.expasy.org/). In our study, the characterized phiAP1 is a temperate phage retaining a circular genome as supported by the possession of integrase.

Our analysis supports that phiAP1 is an active temperate phage, and most widely distributed-type prophage in A. pasteurianus, based on the following: (i) phiAP1 has the ability to infect its cured C-27 strain, (ii) phiAP1-like elements are found in many species of $A$. pasteurianus with similar gene composition and arrangement, (iii) the integrases have a high similarity to each other, and (iv) the sequences of $a t t B-L$ and $a t t B-R$ are conserved. In the five phiAP1-like elements (Fig. 2B), the proteins encoded in the central region are phage-related proteins, and many of them are found in other phage genomes, whereas many short ORFs flanked by the integrase gene are short compared to their bacterial homologs. There is a possibility that the short ORFs are remaining junk DNA generated during infection. Generally, it is known that phage genomes retain a mosaic structure consisting of the different origin phage genome, generating a high diversity of phages in nature ${ }^{23}$. The high conservation of gene composition and arrangement in the phiAP1-like elements supports that phiAP1s have an identical origin during evolutionary history.

Our study revealed that the site-specific integration of the phiAP1 genome into the host attB site was reproducible when phiAP1 was co-cultured with the C-27 strain. Meanwhile, plaque formation by phiAP1 was never observed under any culture condition used in this study. This indicates that phiAP1 is immediately and stably integrated into the host genome after infection. In $\lambda$ phage in $E$. coli, CI repressor protein belonging to the Xre family inhibits the lytic pathway maintaining the prophage state ${ }^{24}$. Pfam search predicted that ORF11, 12, 17, 18 of phiAP1 belongs to the Xre family, which suggests a possibility that these ORFs may control the switching between lytic and lysogenic states. The expression level of these ORFs could be involved in the inability of plaque formation by phiAP1.

The attB site of $A$. pasteurianus NBRC 109446 was suggested as 5'-CACCCCATCCGCCAACTATACTTC-3' with a 24 bp length. A similar sequence with identical length was also conserved in the phiAP1-like element of A. pasteurianus LMG 1262 (Fig. 5). In contrast, other A. pasteurianus strains retain short sequences of the attB site. The difference in $a t t B$ recognition might be caused by integrase amino acid sequence differences; however, the integrases encoded in the phiAP1-like elements have a high similarity to each other, except for the end of the C-terminus. Namely, the integrases of A. pasteurianus NBRC 109446 and LMG 1262 retain an identical extended 21 amino acid sequence in the C-terminus, while other integrases have no extended region (Fig. S3). This suggests that the extended sequence is involved in the recognition of the long attB sequence. Further study is required for understanding the recognition mechanism of phiAP1 integrases. 
Transformation efficiency of a chromosome-integrative vector p2096int in the three Acetobacter strains is sufficient for the general cloning experiment (Table 2). The site-specific recombination of p2096int into the attB site was also reproducibly confirmed. However, an expansion of host range is required to improve the versatility in $\mathrm{AAB}$ group bacteria. In $\lambda$ phage, it is known that CI repressor serves as an immune mechanism for preventing multiple phage infections. This suggests that the CI-like repressors, ORF11, 12, 17, 18, encoded on the phiAP1 genome, negatively acts on the expression or function of phiAP1 integrase to prevent the integration of p2096int into the chromosome; this may be a reason why the parental strain of A. pasteurianus NBRC 109446 was unacceptable for p2096int. However, A. pasteurianus NBRC 3188, an acceptable strain for p2096int, retains three CI-like repressors, ORF10, 16, 17, in the phiAP-like elements. In another p2096int-acceptable A. pasteurianus ATCC 23754, a phiAP-like element is not found in the $t R N A$ locus, suggesting that this strain is non-lysogenic. Further molecular genetic analysis on the three acceptable strains, and improvement of p2096int such as replacement of integrase promoter with a constitutive one will help to understand the host-specificity of the p2096int vector, and contribute to improving the versatility of the vector.

In this study, we also obtained the prophage cured strain, C-27 in A. pasteriuanus NBRC 109446. We believe that the C-27 strain is a useful host based on the following reasons: (i) The strain is "prophage-free safety host" because phage-induced cell lysis does not occur when DNA is damaged. (ii) The cured strain has an equal ability in acetic acid fermentation with the wild-type strain (unpublished data). (iii) If the p2096int vector is modified to a cosmid, it can carry large gene clusters for secondary metabolite biosynthesis. Therefore, the C-27 strain could be a host for the stable production of useful metabolites. This strain, together with the p2096int vector, can be widely used in basic and applied research.

\section{Materials and methods}

Bacteria, plasmids, oligonucleotides, and culture media. Acetic acid bacteria (AAB) used in this study are listed in Table S2, and were obtained from culture collections including JCM, NBRC, IAM, NRIC, ATCC, and DSM. Escherichia coli HST08 for general cloning host, pUC19 as a cloning vector, and pMD19 as a TA cloning vector were purchased from Takara Bio Inc. (Shiga, Japan). An ampicillin-resistant pMV24 plasmid was used as an Acetobacter spp.-E. coli shuttle vector ${ }^{25}$. Oligonucleotide primers used for PCR are summarized in Table S5. AABs were grown at $30^{\circ} \mathrm{C}$ in YPG medium [containing (per liter): yeast extract, 5 g; hipolypeptone, $3 \mathrm{~g}$; glucose, $30 \mathrm{~g}$ ]. E. coli was grown in Luria-Bertani (LB) medium. For preparation of solid medium, 1.5\% agar was added. To select transformants of E. coli and Acetobacter spp., ampicillin was added at $40 \mu \mathrm{g} / \mathrm{mL}$. All chemicals and enzymes used were obtained from Wako Pure Chemical (Osaka, Japan) and Takara Bio Inc., respectively, unless otherwise indicated.

Selection of lysogenic AAB stains. To select lysogens from a total of $177 \mathrm{AAB}$ strains (Table S2) obtained from culture collections, AABs were grown on YPG solid medium containing 0.2 to $4.0 \mu \mathrm{g} / \mathrm{mL}$ mitomycin C (MMC) at $28^{\circ} \mathrm{C}$ for 4 days. AAB strains exhibiting MMC sensitivity on solid medium were subjected to secondary selection with YPG liquid medium containing 0.2 to $4.0 \mu \mathrm{g} / \mathrm{mL}$ MMC with shaking culture at $110 \mathrm{rpm}$. AAB strains in which growth was inhibited by MMC were regarded as candidates for lysogens.

Preparation of fractions containing temperate phages. To observe temperate phages induced from the MMC-treated AABs, phage-containing fractions were prepared by concentrating the supernatant of $A A B$ culture broth. All of the tested AABs were cultured in YPG liquid medium at $30^{\circ} \mathrm{C}$ overnight with shaking at $160 \mathrm{rpm} .1 \mathrm{~mL}$ of the pre-cultured broth was inoculated to $100 \mathrm{~mL}$ of YPG liquid medium supplemented with $4.0 \mathrm{\mu g} / \mathrm{mL}$ MMC in a $500 \mathrm{ml}$ Erlenmeyer flask with baffle. Culture was performed at $30^{\circ} \mathrm{C}$ for $48 \mathrm{~h}$ in a rotary shaker at $160 \mathrm{rpm}$. After removal of the grown cells by centrifugation and filtration with $0.22 \mu \mathrm{m}$ pore size filter, $25 \mathrm{~mL}$ of $30 \%$ polyethylene glycol (PEG) 8,000 solution containing $5 \mathrm{M} \mathrm{NaCl}$ was added to the supernatant, and then incubated at $4{ }^{\circ} \mathrm{C}$ overnight. The samples were transferred into 40PA centrifuge tubes (Hitachi), and centrifuged at $30,000 \mathrm{rpm}$ at $4{ }^{\circ} \mathrm{C}$ for $2 \mathrm{~h}$ with himac $\mathrm{CP} 100 \mathrm{MX}$ ultracentrifuge. After removing the supernatant, the precipitant was suspended with $100-200 \mu \mathrm{L}$ of $0.85 \%$ saline solution or distilled water, resulting in a 500 to 1000 -fold concentration compared to the initial culture broth.

Visualization of phages with TEM analyses. Phage-containing fraction $(3 \mu \mathrm{L})$ was mixed with the same volume of two-fold diluted EM stainer with distilled water (Nisshin-EM. Co., Ltd., Tokyo, Japan), and incubated for $1 \mathrm{~min}$ at room temperature for negative staining. The mixture was spotted on collodion membrane 200-mesh (Nisshin-EM), and incubated for $90 \mathrm{secs}$ at room temperature. Excess staining solution was removed with whatman filter paper. Specimens were examined with a JEM-1010 (JEOL Ltd., Tokyo, JAPAN) operating at $100 \mathrm{kV}$ according to manufacturer's instruction, and micrographs were developed on ELESCOPE FG film $8.2 \times 11.8 \mathrm{~cm}$ (Fujifilm, Tokyo, Japan).

Determination of whole genome sequences of lysogenic phages and bioinformatics analyses. The phage fractions derived from A. pasteurianus NBRC 109446, A. orleanensis NBRC 3170, Acetobacter sp. ATCC 21760, K. xylinus NBRC 13772, K. xylinus NBRC 13773, and K. maltaceti NBRC 14815 were prepared as described above. The fractions were treated with $20 \mu \mathrm{g} / \mathrm{mL}$ DNase I (Sigma-Aldrich Corp, St. Louis, MO, USA) and $10 \mu \mathrm{g} / \mathrm{mL}$ RNase (Sigma-Aldrich) at $37^{\circ} \mathrm{C}$ for $30 \mathrm{~min}$. Their phage genomic DNAs were purified with Phage DNA isolation kit (Norgen Biotek Corp., Ontario, Canada) according to manufacturer's instruction. Quality and quantity of the purified phage genomic DNAs were verified with GeneQuant 100 (GE Healthcare UK Ltd, Buckinghamshire, England), and agarose gel electrophoresis analyses. Genome sequence analysis with next generation sequencing was performed using Illumina MiSeq with 150-bp paired-end reads by Hokkaido System 
Science Co. Ltd. (Sapporo, Japan). DFAST ${ }^{26}$, RAST ${ }^{27}$, 2ndFind (http://biosyn.nih.go.jp/2ndfind/), PHAST ${ }^{15}$ was used for the annotation of phage genomes. Genetyx ver. 13 (GENETYX Corporation, Tokyo, JAPAN) was used for local BLAST searches using draft genome sequences. Schematic representation of gene organization was drawn with drawGeneArrows3 (http://www.ige.tohoku.ac.jp/joho/index.html). The prediction of the $t R N A$ gene was performed with tRNAscan-SE ${ }^{28}$. Multiple alignment was carried out by CLUSTAL W ${ }^{29}$.

Accession numbers. The nucleotide sequences determined in this study have been registered to GenBank under the accession numbers LC644972 for phiAP1 of A. pasteurianus NBRC 109446, LC644971 for phiAO1 of A. orleanensis NBRC 3170, LC644973 for phiAX1 of Acetobacter sp. ATCC 21760, LC644975 for phiKX1 of K. xylinus NBRC 13772, LC644976 for phiKX2 of K. xylinus NBRC 13773, and LC644974 for phiKM1 of K. maltaceti NBRC 14815.

Construction of a phiAP1-cured C-27 strain in A. pasteurianus NBRC 109446. The wild-type strain of $A$. pasteurianus NBRC 109446 was grown in YPG liquid medium containing $0.05 \%$ to $0.1 \%$ Tween 80 at $28{ }^{\circ} \mathrm{C}$ with shaking at $160 \mathrm{rpm}$ for 16 to $24 \mathrm{~h}$. The propagated cells were diluted with sterile distilled water, and spread on YPG solid medium containing MMC to form single colonies. To identify the prophage-cured stains, the prophage region of phiAP1 was detected by colony PCR with primer set M30-ck3F/M30-ck3R, which anneals to the inner region of the phiAP1 genome. To confirm whether the cured strain lost the prophage, boundary regions between host and prophage genomes were analyzed by PCR using primer set M30-ck1F/M30ck1R and M30-ck2F/M30-ck2R.

To construct p2096int-M1, p2096int-M2, and p2096int-M3, inverse PCR was used. Each DNA fragment containing a full length region was amplified by inverse PCR with primer pair, 2096int-M-R/2096int-M1-L for p2096int-M1, 2096int-M-R/2096int-M2-L for p2096int-M2, and 2096int-M-R/2096int-M3-L for p2096int-M3. The purified amplicons were self-ligated with T4 DNA Ligase. The constructed vectors were sequenced by an ABI3100 sequencer (Thermo Fisher Scientific) or Eurofins Genomics K.K. (Tokyo, Japan). The constructed p2096int derivatives were introduced into A. pasteurianus C-27 strain by electroporation as described above.

Construction of ampicillin-resistant lysogenic host of $\boldsymbol{A}$. pasteurianus NBRC 109446 . To construct $A$. pasteurianus NBRC 109446 retaining an ampicillin-resistant gene in the prophage region, an ampicillin(Amp)-resistant pGMS3 vector harboring the 1,365 bp region homologous with the internal sequences of a gene encoding a putative serine peptidase (ORF37 in Fig. 3 and Table S4) was constructed as follows. The DNA fragment containing the internal region of ORF37 gene within phiAP1 was generated by PCR with primer set Dis19188F/Dis20547R. The amplicon was then directly cloned into an Amp-resistant TA vector pMD19, yielding the pGMS3 vector. A. pasteurianus NBRC 109446 wild-type was transformed with pGMS3 by electroporation, and the recovery culture allowing the expression of Amp-resistant gene was performed at $30{ }^{\circ} \mathrm{C}$ for $6 \mathrm{~h}$. The single-crossover strains exhibiting Amp resistance were selected on YPG solid medium containing $40 \mu \mathrm{g} / \mathrm{mL}$ ampicillin. The proper single-crossover recombination to the $a t t B$ site was confirmed by PCR with an appropriate primer set. The constructed strain was designated as the A. pasteurianus GMS3 strain, and its Ampresistant gene-retaining phage was designated as phiAP1-Amp.

Infection of the phiAP1-cured C-27 strain with phiAP1. To prepare the phiAP1-Amp-containing fraction, A. pasteurianus GMS3 strain was cultured in the presence of MMC, and the cell-free supernatant of culture broth was concentrated with $30 \%$ PEG 8,000 solution containing $5 \mathrm{M} \mathrm{NaCl}$ as described above. The phiAP1-Amp-containing fraction was spotted onto the lawn of the C-27 strain grown on YPG solid medium, and the plates were incubated at $30^{\circ} \mathrm{C}$ for $48 \mathrm{~h}$. The C-27 strain grown in the phage-spotted region was harvested with a spatula, and the cell suspended in sterile distilled water was inoculated onto YPG solid medium containing $40 \mu \mathrm{g} / \mathrm{mL}$ ampicillin.

Construction of a chromosome-integrative vector p2096int for A. pasteurianus C-27 strain. A $1,210 \mathrm{bp}$ DNA fragment containing attP, a promoter region of integrase, as well as the $\mathrm{N}$-terminal region of integrase, was amplified by PCR with primer set 2096int-F1/2096int-R1. The 863 bp DNA fragment containing the C-terminal region of the integrase gene was amplified by PCR with 2096int-F2/2096int-R2 using a synthetic DNA of C-terminal region of the integrase gene as template. An $840 \mathrm{bp}$ DNA fragment containing the attP and integrase gene of phiAP1 was synthesized by Eurofins Genomics K.K. (Tokyo, Japan) to remove the restriction enzyme sites used in the multiple cloning site. The above two DNA fragments were fused by overlapping PCR using primer set 2096int-F1/2096int-R2. The amplicon was cleaved with NdeI, and inserted into the same site of pUC19, yielding a p2096int that was 4,723 bp in total length. The constructed p2096int was introduced into AAB strains by electroporation. Transformants were selected on YPG solid medium containing $40 \mu \mathrm{g} / \mathrm{mL} \mathrm{Amp}$, and proper integration was confirmed by PCR with an appropriate primer set.

Determination of attB sites in A. pasteurianus NBRC 3188 and A. pasteurianus subsp. pasteurianus ATCC 23754. The attB site of $A$. pasteurianus NBRC 3188 was predicted by homology search with the draft genomic information (GenBank accession number: NZ_BDES00000000) using local BLAST in Genetyx. To predict the nucleotide sequence of the attB site in A. pasteurianus ATCC 23754, the intergenic regions of Elongation factor P and the upstream ORF66 were amplified by PCR with primer pair, tRNASer_attB_F2/ tRNASer_attB_R4, which were constructed based on nucleotide sequences conserved among Acetobacter spp. The amplicons were cloned into pMD19 vector by TA cloning, and nucleotide sequences of the resultant clones 
were determined by sequencing service of Eurofins Genomics or using an ABI 3100 Genetic analyzer (Thermo Fisher Scientific, Waltham, MA, USA).

Received: 27 July 2021; Accepted: 19 October 2021

Published online: 03 November 2021

\section{References}

1. Saichana, N., Matsushita, K., Adachi, O., Frébort, I. \& Frebortova, J. Acetic acid bacteria: A group of bacteria with versatile biotechnological applications. Biotechnol. Adv. 33, 1260-1271 (2015).

2. De Roos, J. \& De Vuyst, L. Acetic acid bacteria in fermented foods and beverages. Curr. Opin. Biotechnol. 49, 115-119 (2018)

3. Raspor, P. \& Goranovic, D. Biotechnological applications of acetic acid bacteria. Crit. Rev. Biotechnol. 28, 101-124 (2008).

4. Rohwer, F. \& Edwards, R. The Phage Proteomic Tree: A genome-based taxonomy for phage. J. Bacteriol. 184, 4529-4535 (2002).

5. Boyd, E. F. \& Brüssow, H. Common themes among bacteriophage-encoded virulence factors and diversity among the bacteriophages involved. Trends Microbiol. 10, 521-529 (2002).

6. Frost, L. S., Leplae, R., Summers, A. O. \& Toussaint, A. Mobile genetic elements: The agents of open source evolution. Nat. Rev. Microbiol. 3, 722-732 (2005).

7. Smets, B. F. \& Barkay, T. Horizontal gene transfer: Perspectives at a crossroads of scientific disciplines. Nat. Rev. Microbiol. 3 , 675-678 (2005).

8. Williamson, K. E., Schnitker, J. B., Radosevich, M., Smith, D. W. \& Wommack, K. E. Cultivation-based assessment of lysogeny among soil bacteria. Microb. Ecol. 56, 437-447 (2008).

9. Davies, E. V., Winstanley, C., Fothergill, J. L. \& James, C. E. The role of temperate bacteriophages in bacterial infection. FEMS Microbiol. Lett. 363, 15 (2016).

10. Nafissi, N. \& Slavcev, R. Bacteriophage recombination systems and biotechnical applications. Appl. Microbiol. Biotechnol. 98, 2841-2851 (2014).

11. Robakis, N. K. et al. Isolation and Characterization of 2 Phages for Gluconobacter oxydans. J. Gen. Microbiol. 131, 2467-2473 (1985).

12. Kiesel, B. \& Wunsche, L. Phage Acm1-mediated transduction in the facultatively methanol-utilizing Acetobacter methanolicus MB 58/4. J. Gen. Virol. 74, 1741-1745 (1993).

13. Schocher, A. J. et al. Acetobacter bacteriophage A-1. Arch. Microbiol. 121, 193-197 (1979).

14. Philippe, C. et al. Bacteriophage GC1, a novel tectivirus infecting Gluconobacter cerinus, an acetic acid bacterium associated with wine-making. Viruses 10, 39 (2018).

15. Zhou, Y., Liang, Y., Lynch, K. H., Dennis, J. J. \& Wishart, D. S. PHAST: A fast phage search tool. Nucl. Acids Res. 39, W347-W352 (2011).

16. Casjens, S. Prophages and bacterial genomics: What have we learned so far?. Mol. Microbiol. 49, 277-300 (2003).

17. Azuma, Y. et al. Whole-genome analyses reveal genetic instability of Acetobacter pasteurianus. Nucl. Acids Res. 37, 5768-5783 (2009).

18. Kubiak, K. et al. Complete genome sequence of Gluconacetobacter xylinus E25 strain-valuable and effective producer of bacterial nanocellulose. J. Biotechnol. 176, 18-19 (2014).

19. Labrie, S. J., Samson, J. E. \& Moineau, S. Bacteriophage resistance mechanisms. Nat. Rev. Microbiol. 8, 317-327 (2010).

20. Groman, N. B. \& Bobb, D. The inhibition of adsorption of Corynebacterium diphtheriae phage by tween 80. Virology 1,313-323 (1955).

21. Groth, A. C. \& Calos, M. P. Phage integrases: biology and applications. J. Mol. Biol. 335, 667-678 (2004).

22. Ohmori, S. \& Uozumi, T. Loss of acetic acid resistance and ethanol oxidizing ability in an Acetobacter strain. Agric. Biol. Chem. 46, 381-389 (1982).

23. Morris, P., Marinelli, L. J., Jacobs-Sera, D., Hendrix, R. W. \& Hatfull, G. F. Genomic characterization of mycobacteriophage giles: Evidence for phage acquisition of host DNA by illegitimate recombination. J Bacteriol 1, 1 (2008).

24. Casjens, S. R. \& Hendrix, R. W. Bacteriophage lambda: Early pioneer and still relevant. Virology 479-480, 310-330 (2015).

25. Fukaya, M. et al. Cloning of the Membrane-Bound Aldehyde Dehydrogenase Gene of Acetobacter polyoxogenes and Improvement of Acetic Acid Production by Use of the Cloned Gene. Appl. Environ. Microbiol. 55, 171-176 (1989).

26. Lowe, T. M. \& Chan, P. P. tRNAscan-SE On-line: Integrating search and context for analysis of transfer RNA genes. Nucl. Acids Res. 44, W54-W57 (2016).

27. Thompson, J. D., Higgins, D. G. \& Gibson, T. J. CLUSTAL W: Improving the sensitivity of progressive multiple sequence alignment through sequence weighting, position-specific gap penalties and weight matrix choice. Nucl. Acids Res. 22, 4673-4680 (1994).

\section{Acknowledgements}

We wish to thank Mr. Takashi Sone for technical assistant on determination of attB sites.

\section{Author contributions}

N.H, S.N, S.K, K.U, H.T. designed the experiments. K.O, N.H, S.N, K.S, K.N, S.A. performed phage screening and TEM analysis. N.H, S.N. performed genomic sequence analysis. K.O, N.H, S.N. constructed the C-27 strain. K.O. constructed the GMS3 strain and p2096int vector. H.T. performed comparative genome analysis. K.O. took a photo of Fig. 6. H.T. wrote the manuscript. N.H, H.T, and K.U. integrated the overall research project. All authors discussed the results and commented on the manuscript.

\section{Competing interests}

The authors declare no competing interests.

\section{Additional information}

Supplementary Information The online version contains supplementary material available at https://doi.org/ 10.1038/s41598-021-00998-w.

Correspondence and requests for materials should be addressed to H.T.

Reprints and permissions information is available at www.nature.com/reprints. 
Publisher's note Springer Nature remains neutral with regard to jurisdictional claims in published maps and institutional affiliations.

(c) (i) Open Access This article is licensed under a Creative Commons Attribution 4.0 International License, which permits use, sharing, adaptation, distribution and reproduction in any medium or format, as long as you give appropriate credit to the original author(s) and the source, provide a link to the Creative Commons licence, and indicate if changes were made. The images or other third party material in this article are included in the article's Creative Commons licence, unless indicated otherwise in a credit line to the material. If material is not included in the article's Creative Commons licence and your intended use is not permitted by statutory regulation or exceeds the permitted use, you will need to obtain permission directly from the copyright holder. To view a copy of this licence, visit http://creativecommons.org/licenses/by/4.0/.

(C) The Author(s) 2021 